\title{
Regulation of protein synthesis associated with skeletal muscle hypertrophy by insulin-, amino acid- and exercise-induced signalling
}

\author{
Douglas R. Bolster, Leonard S. Jefferson and Scot R. Kimball* \\ Department of Cellular and Molecular Physiology, The Pennsylvania State University College of Medicine, \\ PO Box 850, Hershey, PA 17033, USA
}

\begin{abstract}
Although insulin, amino acids and exercise individually activate multiple signal transduction pathways in skeletal muscle, one pathway, the phosphatidylinositol 3-kinase (PI3K)-mammalian target of rapamycin (mTOR) signalling pathway, is a target of all three. Activation of the PI3K-mTOR signal transduction pathway results in both acute (i.e. occurring in minutes to hours) and long-term (i.e. occurring in hours to days) up-regulation of protein synthesis through modulation of multiple steps involved in mediating the initiation of mRNA translation and ribosome biogenesis respectively. In addition, changes in gene expression through altered patterns of mRNA translation promote cell growth, which in turn promotes muscle hypertrophy. The focus of the present discussion is to review current knowledge concerning the mechanism(s) through which insulin, amino acids and resistance exercise act to activate the PI3K-mTOR signal transduction pathway and thereby enhance the rate of protein synthesis in muscle.
\end{abstract}

Resistance exercise: Phosphatidylinositol 3-kinase: Mammalian target of rapamycin: mRNA translation

A vast array of pathways within mammalian cells serves to transduce signals from external stimuli to processes such as nutrient uptake and metabolism, gene transcription and mRNA translation. In most cases the transduction network consists of a group of protein kinases that serve to direct a signal, or signals, to the appropriate end point(s). However, signal transduction pathways are seldom linear and instead often have many branch points and multiple places at which signalling events can activate the kinase cascade. An example of this type of complexity is exhibited by a common pathway that is activated by the signals induced by insulin, amino acids and resistance exercise, i.e. the phosphatidylinositol 3-kinase (PI3K)-mammalian target of rapamycin (mTOR) signalling pathway. The purpose of the present article is to review current knowledge of how these disparate signals activate this pathway and how signals from the individual stimuli are integrated, with the focus being on how insulin, amino acids and exercise promote muscle hypertrophy through activation of this pathway. As the present article is only a brief overview, the work cited herein is selective. For further discussion on this topic the reader is referred to other recent review articles (for example, see Kimball et al. 2002; Bolster et al. 2003).

\section{The phosphatidylinositol 3-kinase-mammalian target of rapamycin signalling pathway}

PI3K is a lipid kinase that phosphorylates the hydroxyl group at the D3 position on phosphatidylinositol-4, 5-diphosphate leading to the production of phosphatidylinositol-3,4,5-triphosphate at the plasma membrane (for review, see Cantley, 2002). Production of phosphatidylinositol-3,4,5-triphosphate is countered by the action of the lipid phosphatase, phosphatase and tensin homologue (Fig. 1). Phosphatidylinositol-3,4,5-triphosphate recruits proteins containing pleckstrin homology domains, such as phosphatidylinositol-dependent protein kinase and Akt/ protein kinase $\mathrm{B}(\mathrm{PKB})$, to the plasma membrane where they are activated. Akt/PKB serves as a branch point in the PI3K-mTOR signalling pathway and has been reported to phosphorylate glycogen synthase kinase (GSK) $3 \beta$ at $\operatorname{Ser}^{9}\left(\operatorname{Ser}^{23}\right.$ on GSK-3 $\alpha$; for review, see Coffer et al. 1998), mTOR at $\operatorname{Ser}^{2448}$ (Nave et al. 1999) and tuberous sclerosis complex (TSC) 2 on multiple residues including $\mathrm{Ser}^{939}$, $\mathrm{Ser}^{1130}$ and $\mathrm{Thr}^{1462}$ (for review, see Manning \& Cantley, 2003). Phosphorylation of GSK-3 $\beta$ on $\operatorname{Ser}^{9}$ results in its inactivation, an event that leads to dephosphorylation, and thereby activation, of eukaryotic initiation factor (eIF) $2 \mathrm{~B}$

\footnotetext{
Abbreviations: eIF, eukaryotic initiation factor; 4E-BP1, eIF4E-binding protein 1; GSK, glycogen synthase kinase; mTOR, mammalian target of rapamycin; PI3K, phosphatidylinositol 3-kinase; raptor, regulatory associated protein of target of rapamycin; Rheb, ras homologue enriched in brain; S6K1, ribosomal protein S6 protein kinase; TSC, tuberous sclerosis complex.

*Corresponding author: Professor Scot R. Kimball, fax +1717531 7667, email skimball@psu.edu
} 


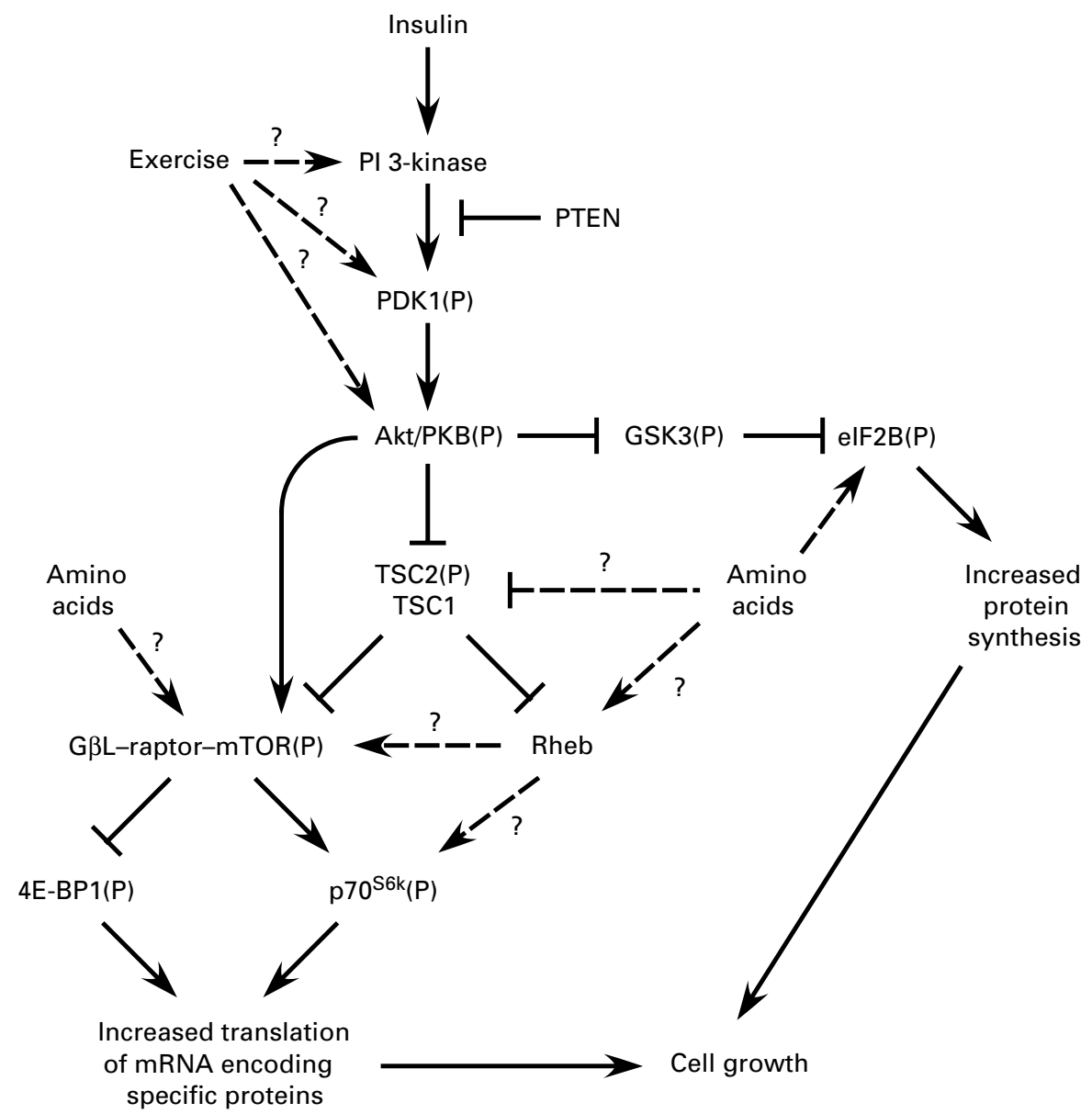

Fig. 1. Components of the phosphatidylinositol (PI) 3-kinase-mammalian target of rapamycin (mTOR) signalling pathway. Insulin, amino acids and exercise have been shown to activate various components of the PI 3-kinase-mTOR signal transduction pathway leading to enhanced muscle protein synthesis and hypertrophy, as described in detail on pp. 351-355. $\longleftarrow, \leftarrow-$, Activating steps; $\longmapsto$, $\vdash--$, inhibitory steps in the pathway. --, Incompletely-defined links. PDK1, phosphatidylinositol-dependent protein kinase 1; P, phosphate; PKB, protein kinase B; GSK3, glycogen synthase kinase 3; elF2B, eukaryotic initiation factor 2B; TSC, tuberous sclerosis complex; raptor, regulatory associated protein of target of rapamycin; Rheb, ras homologue enriched in brain; mTOR, mammalian target of rapamycin protein kinase; 4E-BP1, elF4E-binding protein 1; $\mathrm{p} 70^{\mathrm{S} 6 \mathrm{k}}, 70 \mathrm{kDa}$ ribosomal protein $\mathrm{S} 6$ protein kinase; PTEN, phosphatase and tensin homologue.

(Welsh et al. 1998). TSC2, in a complex with TSC1, normally represses signalling through $\mathrm{MTOR}$, and phosphorylation by Akt/PKB inhibits this function of TSC2 (for review, see Manning \& Cantley, 2003). Although the mechanism through which the TSC1-TSC2 complex represses mTOR signalling is incompletely defined, recent studies (Garami et al. 2003; Zhang et al. 2003) have suggested that, in part, TSC2 may act through the small GTPase ras homologue enriched in brain (Rheb) in regulating mTOR. Here, TSC2 is a GTPase-activating protein for Rheb that normally represses Rheb function (Garami et al. 2003; Zhang et al. 2003). How Rheb modulates mTOR-dependent signalling is unknown. In contrast to the effect of phosphorylation by Akt/PKB on most of its substrates, phosphorylation of mTOR on $\mathrm{Ser}^{2448}$ reportedly promotes mTOR-dependent signalling (Nave et al. 1999). Activation of mTOR results in phosphorylation of a variety of substrates, including the eIF4E-binding protein (4E-BP1), and the ribosomal protein S6 protein kinase (S6K1; for review, see Gingras et al. 2001). Phosphorylation of both substrates requires the interaction of mTOR with two other proteins, the regulatory associated protein of target of rapamycin (raptor) and GßL (Kim et al. 2003).

\section{Regulation of mRNA translation through activation of the phosphatidylinositol 3-kinase-mammalian target of rapamycin signalling pathway}

The mechanisms involved in the stimulation of protein synthesis that occurs in response to activation of the PI3K-mTOR signalling pathway can be temporally separated into two categories. Rapid changes, i.e. those occurring in $<1 \mathrm{~h}$, can be traced to enhanced rates of 
mRNA translation mediated by activation of translation initiation factors. In contrast, longer-term changes, i.e. those occurring after several hours, are typically a result of an increase in the number of ribosomes available to translate mRNA (i.e. an increase in the capacity to synthesize protein).

One translation initiation factor that is rapidly activated through the PI3K-mTOR signalling pathway is eIF2B, a guanine nucleotide exchange factor for a second initiation factor, eIF2 (for review, see Hershey \& Merrick, 2000). During the first step in the initiation of mRNA translation, eIF2 binds to the $40 \mathrm{~S}$ ribosomal subunit as a ternary complex with GTP and initiator methionyltRNA. Subsequently, the GTP bound to eIF2 is hydrolysed and eIF2-GDP is released from the ribosome. eIF2B mediates exchange of GDP bound to eIF2 for free GTP, allowing the ternary complex to reform. As the synthesis of all proteins begins with initiator methionyltRNA, activation of eIF2B globally stimulates protein synthesis.

Activation of the PI3K-mTOR signalling pathway also results in the rapid phosphorylation of 4E-BP1 and S6K1. 4E-BP1 binds to the mRNA cap-binding protein eIF4E and prevents it from binding to a second initiation factor eIF4G (for review, see Hershey \& Merrick, 2000). Phosphorylation of 4E-BP1 results in its release from eIF4E, allowing eIF4E to bind to eIF4G to form the active eIF4F complex that mediates binding of mRNA to the $40 \mathrm{~S}$ ribosomal subunit. Enhanced eIF4F assembly results in a preferential increase in the translation of mRNA with long highlystructured 5'-untranslated regions, such as those encoding ornithine decarboxylase and cyclin D1. Overexpression of eIF4E increases cell size, presumably through enhanced assembly of the active eIF4F complex, whereas exogenous expression of a non-phosphorylatable form of 4E-BP1 results in a small cell phenotype (Fingar et al. 2002), demonstrating the importance of these two proteins in controlling cell growth.

Activation of S6K1 also occurs within minutes of initiation of signalling through mTOR. S6K1 phosphorylates ribosomal protein $\mathrm{S6}$, which reportedly enhances the translation of mRNA with an uninterrupted string of pyrimidine residues adjacent to the 5'-cap structure (terminal oligopyrimidine mRNA; Meyuhas, 2000). Proteins encoded by such mRNA include ribosomal proteins, translation elongation factors and poly(A) binding protein. Thus, activation of S6K1 results in a rapid increase in the synthesis of a subset of proteins. In addition, mTOR signalling enhances the transcription of ribosomal DNA. Together, increased translation of mRNA encoding ribosomal proteins and enhanced transcription of ribosomal DNA promotes ribosome biogenesis. An increase in the cellular content of ribosomes is one mechanism for increasing the long-term capacity of the cell to synthesize protein. Similar to the effect of eIF4E overexpression, exogenous expression of S6K1 increases cell size (Fingar et al. 2002). Interestingly, coexpression of eIF4E and S6K1 cooperatively increases cell size, suggesting that the events controlled by the two proteins function independently to modulate cell growth.

\author{
Activation of the phosphatidylinositol \\ 3-kinase-mammalian target of rapamycin \\ signalling pathway by insulin
}

The first step in the activation of the PI3K-mTOR signalling pathway by insulin is binding of the hormone to its receptor, resulting in activation of the receptor's intrinsic tyrosine protein kinase activity (for review, see Alessi \& Downes, 1998). The activated insulin receptor subsequently phosphorylates the insulin receptor substrates 1 and 2. The phosphorylated insulin receptor substrates bind to the $85 \mathrm{kDa}$ subunit of PI3K, thereby activating the kinase and the PI3K-mTOR signalling pathway. An intermediate in the pathway, PKB (or Akt) acts as a branch point and phosphorylates both mTOR and GSK-3. Phosphorylation of GSK-3 by PKB results in its inactivation. GSK-3 phosphorylates the $\varepsilon$ subunit of eIF2B, which is associated with decreased guanine nucleotide exchange activity in cells in culture (Welsh et al. 1998) and therefore represents one potential mechanism through which insulin regulates protein synthesis. Activation of mTOR by insulin results in phosphorylation of downstream targets, e.g. 4E-BP1, S6K1 and ribosomal protein S6, resulting in activation of the mRNA-binding steps in translation initiation.

\section{Activation of the phosphatidylinositol 3-kinase-mammalian target of rapamycin signalling pathway by amino acids}

Although it is clear that amino acids, and in particular leucine, enhance phosphorylation of proteins downstream of mTOR, e.g. 4E-BP1 and S6K1, and indeed require mTOR to be active in order to be effective, whether or not they directly regulate mTOR activity is undecided. This point is particularly important because, unlike insulin, amino acids do not activate PI3K or PKB (for example, see Hara et al. 1998; Patti et al. 1998). Instead of signalling through Akt, recent studies suggested that amino acids may indirectly modulate mTOR-dependent signalling through TSC1-TSC2, GßL-raptor and/or Rheb. For example, repressed expression of TSC2 (Gao et al. 2002) yields cells that are resistant to the effects of amino acid deprivation on signalling events downstream of mTOR. In contrast, overexpression of the TSC1-TSC 2 complex (Tee et al. 2002) or reduced expression of Rheb (Saucedo et al. 2003) repress signalling downstream of mTOR even in the presence of amino acids. Finally, amino acids promote the association of raptor with mTOR, an effect that requires $\mathrm{G} \beta \mathrm{L}$, thereby enhancing phosphorylation of 4E-BP1 and S6K1 (Kim et al. 2003).

\section{Activation of the phosphatidylinositol 3-kinase-mammalian target of rapamycin signalling pathway by resistance exercise}

Unlike changes noted with intracellular signalling in response to insulin stimulation, skeletal muscle loading does not always lead to comparable alterations in the PI3K-mTOR signalling pathway. For example, no studies to date have reported upstream changes in PI3K activity with resistance exercise during the early phases of recovery, 
although one report has suggested a delayed response whereby increases are revealed $6 \mathrm{~h}$ post exercise (Hernandez et al. 2000). Moreover, the immediate downstream effector of PI3K, phosphatidylinositol-dependent protein kinase 1, has not been examined following muscle contraction or loading conditions. A study by Bodine et al. (2001) has provided the most convincing data supporting a direct role for Akt/mTOR signalling in regulating mRNA translation and promoting skeletal muscle hypertrophy. The study showed that chronic overloading of the plantaris (through synergistic ablation of the soleus and gastrocnemius) leads to increased phosphorylation of $\mathrm{Akt} / \mathrm{PKB}$ and proteins downstream of mTOR, such as 4E-BP1 and S6K1. The central role of mTOR in mediating the hypertrophic response under these loading conditions has been verified through in vivo treatment with rapamycin (a specific inhibitor of mTOR), which almost completely blocks the muscle hypertrophy associated with synergistic ablation. A second study using the synergistic ablation model has reported that overload promotes phosphorylation of $\mathrm{Ser}^{2448}$ on mTOR (Reynolds et al. 2002), a result that is consistent with activation of Akt/PKB. Recently published data have revealed rapid up-regulation of Akt activity in isolated skeletal muscle subjected to passive stretch, which appears specific to fast-twitch muscle (Sakamoto et al. 2003). The stretch-induced activation of Akt is completely blocked through pretreatment with wortmannin, an inhibitor of PI3K. In other studies phosphorylation of both 4E-BP1 and S6K1 has been shown to be enhanced in response to muscle loading (Baar \& Esser, 1999; Bodine et al. 2001; Nader \& Esser, 2001; Rommel et al. 2001) and positively contributes to the up-regulation of select gene expression necessary to elicit long-term increases in skeletal muscle accretion. Indeed, each of these select proteins is indispensable in mediating skeletal muscle growth and, whereas proposing the up-regulation of distinct proteins as potential markers of hypertrophy is tempting, a coordinated response from multiple signal transduction pathways is essential to the overall process.

\section{Integration of signalling by insulin, amino acids and resistance exercise}

A thorough review of amino acids and insulin and their contribution to regulating skeletal muscle protein synthesis is beyond the scope of the present paper. Thus, the intent of the present brief overview will be to focus exclusively on the impact of amino acids or insulin on protein synthesis in the context of resistance exercise. Without question, investigating the singular role of amino acids or insulin in promoting changes in skeletal muscle protein synthesis with resistance exercise is crucial to elucidating mechanisms regulating muscle hypertrophy. However, in a physiological state the concept is essentially irrelevant, as insulin and amino acids do not function in isolation because both participate to optimize the anabolic response in skeletal muscle. Nonetheless, key findings in both areas will be briefly highlighted to emphasize their contribution with resistance exercise.

Although insulin concentrations remain relatively unchanged in the recovery period following resistance exercise (Kraemer et al. 1998; Biolo et al. 1999; Hernandez et al. 2000), a permissive amount of insulin is required to mediate increases in skeletal-muscle protein synthesis. This concept is illustrated by the results of studies utilizing diabetic rats in which animals engaged in resistance exercise (Farrell et al. 1999; Fedele et al. 2000; Kostyak et al. 2001). In such studies rats are operantly conditioned to touch an illuminated bar high on a Plexiglas exercise cage while wearing a weighted vest strapped over the scapulae (Fluckey et al. 1995). The movement performed during the exercise requires the animals to complete concentric and eccentric muscle contractions of the hindlimb musculature (Fluckey et al. 1995). While moderatelydiabetic animals (fed arterial insulin, approximately 180 pM) exhibit an increase in protein synthesis rate as well as augmented eIF2B activity post-exercise, severely-diabetic (fed arterial insulin, approximately 72 pM) animals exhibit depressed protein synthesis rates and eIF2B activity at rest, which fails to change with subsequent exercise (Farrell et al. 1999; Fedele et al. 2000; Kostyak et al. 2001). In contrast with the results of studies using rodents, elevated insulin concentrations induced through local infusions in human subjects have been shown to stimulate skeletal muscle protein synthesis at rest, but hyperinsulinaemia fails to further augment synthesis after resistance exercise (Biolo et al. 1999). Collectively, this body of evidence suggests an endocrine threshold for insulin when coupled with resistance exercise, whereby protein synthesis remains refractory when plasma levels above or below this range are achieved.

The kinetics of amino acid metabolism with resistance exercise has been extensively examined. The incorporation of stable-isotope methodology along with skeletal muscle biopsies has greatly improved the understanding of the in vivo effects of alterations in amino acid transport and amino acid availability on skeletal muscle protein synthesis with resistance exercise. Biolo et al. (1995a) have impressively demonstrated marked increases in the delivery of amino acids to the leg $3 \mathrm{~h}$ after resistance exercise in a post-absorptive state. The influx of amino acids from the artery to the muscle is preferentially enhanced for lysine, leucine and alanine. Furthermore, these studies provide evidence for the unique synergy between amino acid availability, blood flow and the stimulus of physical exercise to maximize increases in protein synthesis (Biolo et al. 1997).

The issue of nutrient availability is worth noting, given the tremendous interest in dietary supplementation among exercising individuals. The issue of timing nutrient intake has received intense scrutiny, and data suggest that supplying amino acids plus glucose either before exercise or at various intervals during recovery enhances skeletal-muscle protein synthesis (Rasmussen et al. 2000; Tipton et al. 2001). However, the magnitude of the protein synthetic response is greatest when nutrients are received immediately before exercise. The basic tenet of consuming adequate nutrients with resistance exercise is reinforced with this area of research, such that a positive net balance is achieved only when exogenous nutrients are available. This positive balance in turn enhances protein synthesis, attenuates breakdown and promotes an anabolic 
environment during the recovery period (Biolo et al. $1995 b)$.

\section{Conclusions}

Insulin, amino acids and resistance exercise all lead to enhancements in protein synthesis in skeletal muscle. A common end point in signalling by each stimulus is the protein kinase mTOR. Inhibition of mTOR by rapamycin or through genetic means (e.g. using RNA interference in cells in culture) prevents the increase in protein synthesis caused by any of the three stimuli. Moreover, rapamycin dramatically attenuates the hypertrophy observed in the synergistic ablation model of resistance exercise. In fact, both in cells in culture and in animals in vivo, inhibition of mTOR results in a small cell phenotype. Overall, the available evidence strongly suggests a central role for mTOR in controlling cell growth. Now that the important role played by mTOR in hypertrophy has been identified, future studies should soon provide more details concerning the mechanism through which amino acids and resistance exercise promote signalling through this kinase. In addition, linking downstream events induced by mTOR activation to subsequent changes in gene expression will need to be addressed in future studies. Together, studies focusing both upstream and downstream of mTOR will lead to a better understanding of how insulin, amino acids and resistance exercise enhance protein synthesis and hypertrophy in skeletal muscle.

\section{Acknowledgements}

The work described in the present article, which was performed in the authors' laboratories, was supported by grant DK15658 from the National Institutes of Health. D.R.B. is supported by a Mentor Based Postdoctoral Fellowship grant from the American Diabetes Association (awarded to L.S.J.).

\section{References}

Alessi DR \& Downes CP (1998) The role of PI 3-kinase in insulin action. Biochimica et Biophysica Acta 1436, 151-164.

Baar K \& Esser KA (1999) Phosphorylation of p70 ${ }^{\text {S6k }}$ correlates with increased skeletal muscle mass following resistance exercise. American Journal of Physiology 276, C120-C127.

Biolo G, Fleming R \& Wolfe R (1995a) Physiologic hyperinsulinemia stimulates protein synthesis and enhances transport of selected amino acids in human skeletal muscle. Journal of Clinical Investigation 95, 811-819.

Biolo G, Maggi S, Williams B, Tipton K \& Wolfe R (1995b) Increased rates of muscle protein turnover and amino acid transport following resistance exercise in humans. American Journal of Physiology 268, E514-E520.

Biolo G, Tipton KD, Klein S \& Wolfe RR (1997) An abundant supply of amino acids enhances the metabolic effect of exercise on muscle protein. American Journal of Physiology 273, E122-E129.

Biolo G, Williams BD, Fleming RY \& Wolfe RR (1999) Insulin action on muscle protein kinetics and amino acid transport during recovery after resistance exercise. Diabetes 48, 949-957.
Bodine SC, Stitt TN, Gonzalez M, Kline WO, Stover GL, Bauerlein R, Zlotchenko E, Scrimgeour A, Lawrence JC, Glass DJ \& Yancopoulous GD (2001) Akt/mTOR pathway is a crucial regulator of skeletal muscle hypertrophy and can prevent muscle atrophy in vivo. Nature Cell Biology 3, 1014-1019.

Bolster DR, Kimball SR \& Jefferson LS (2003) Translational control mechanisms modulate skeletal muscle gene expression during hypertrophy. Exercise and Sport Sciences Reviews 31, 111-116.

Cantley LC (2002) The phosphoinositide 3-kinase pathway. Science 296, 1655-1657.

Coffer PJ, Jin J \& Woodgett JR (1998) Protein kinase B (c-Akt): a multifactorial mediator of phosphatidylinositol 3-kinase activation. Biochemical Journal 335, 1-13.

Farrell PA, Fedele MJ, Vary TC, Kimball SR, Lang CH \& Jefferson LS (1999) Regulation of protein synthesis after acute resistance exercise in diabetic rats. American Journal of Physiology 276, E721-E727.

Fedele MJ, Hernandez JM, Lang CH, Vary TC, Kimball SR, Jefferson LS \& Farrell PA (2000) Severe diabetes prohibits elevations in muscle protein synthesis after acute resistance exercise in rats. Journal of Applied Physiology 88, 102-108.

Fingar DC, Salama S, Tsou C, Harlow E \& Blenis J (2002) Mammalian cell size is controlled by mTOR and its downstream targets S6K1 and 4EBP1/eIF4E. Genes and Development 16, 1472-1487.

Fluckey JD, Kraemer WJ \& Farrell PA (1995) Pancreatic islet insulin secretion is increased after resistance exercise in rats. Journal of Applied Physiology 79, 1100-1105.

Gao X, Zhang Y, Arrazola P, Hino O, Kobayashi T, Yeung RS, $\mathrm{Ru}$ B \& Pan D (2002) Tsc tumour suppressor proteins antagonize amino acid-mTOR signaling. Nature Cell Biology 4, 699-704.

Garami A, Zwartkruis FJT, Nobukuni T, Joaquin M, Roccio M, Stocker H, Kozma SC, Hafen E, Bos JL \& Thomas G (2003) Insulin activation of Rheb, a mediator of mTOR/S6K/4E-BP1 signaling, is inhibited by TSC1 and 2. Molecular Cell 11, $1457-1466$.

Gingras A-C, Raught B \& Sonenberg N (2001) Regulation of translation initiation by FRAP/mTOR. Genes and Development 15, 807-826.

Hara K, Yonezawa K, Weng Q-P, Kozlowski MT, Belham C \& Avruch J (1998) Amino acid sufficiency and mTOR regulate p70 S6 kinase and eIF-4E BP1 through a common effector mechanism. Journal of Biological Chemistry 273, 14484-14494.

Hernandez JM, Fedele MJ \& Farrell PA (2000) Time course evaluation of protein synthesis and glucose uptake after acute resistance exercise in rats. Journal of Applied Physiology 88, $1142-1149$.

Hershey JWB \& Merrick WC (2000) The pathway and mechanism of initiation of protein synthesis. In Translational Control of Gene Expression, pp. 33-88. [N Sonenberg, JWB Hershey and MB Mathews, editors]. Cold Spring Harbor, NY: Cold Spring Harbor Laboratory Press.

Kim D-H, Sarbassov DD, Ali SM, Latek RR, Guntur KVP, Erdjument-Bromage H, Tempst P \& Sabatini D (2003) GßL, a positive regulator of the rapamycin-sensitive pathway required for the nutrient-sensitive interaction between raptor and mTOR. Molecular Cell 11, 895-904.

Kimball SR, Farrell PA \& Jefferson LS (2002) Role of insulin in translational control of protein synthesis in skeletal muscle by amino acids or exercise. Journal of Applied Physiology 93, $1168-1180$.

Kostyak JC, Kimball SR, Jefferson LS \& Farrell PA (2001) Severe diabetes inhibits resistance exercise-induced increase in eukaryotic initiation factor 2B activity. Journal of Applied Physiology 91, 79-84. 
Kraemer WJ, Volek JS, Bush JA, Putukian M \& Sebastianelli WJ (1998) Hormonal responses to consecutive days of heavyresistance exercise with or without nutritional supplementation. Journal of Applied Physiology 85, 1544-1555.

Manning BD \& Cantley LC (2003) United at last: the tuberous sclerosis complex gene products connect the phosphoinositide 3-kinase/Akt pathway to mammalian target of rapamycin (mTOR) signalling. Biochemical Society Transactions 31, 573-578.

Meyuhas O (2000) Synthesis of the translational apparatus is regulated at the translational level. European Journal of Biochemistry 267, 6321-6330.

Nader GA \& Esser KA (2001) Intracellular signaling specificity in skeletal muscle in response to different modes of exercise. Journal of Applied Physiology 90, 1936-1942.

Nave BT, Ouwens DM, Withers DJ, Alessi DR \& Shepherd PR (1999) Mammalian target of rapamycin is a direct target for protein kinase B: Identification of a convergence point for opposing effects of insulin and amino acid deficiency on protein translation. Biochemical Journal 344, 427-431.

Patti M-E, Brambilla E, Luzi L, Landaker EJ \& Kahn CR (1998) Bidirectional modulation of insulin action by amino acids. Journal of Clinical Investigation 101, 1519-1529.

Rasmussen BB, Tipton KD, Miller SL, Wolf SE \& Wolfe RR (2000) An oral essential amino acid-carbohydrate supplement enhances muscle protein anabolism after resistance exercise. Journal of Applied Physiology 88, 386-392.

Reynolds TH, Bodine SC \& Lawrence JC (2002) Control of $\mathrm{Ser}^{2448}$ phosphorylation in the mammalian target of rapamycin by insulin and skeletal muscle load. Journal of Biological Chemistry 277, 17657-17662.
Rommel C, Bodine SC, Clarke BA, Rossman R, Nunez L, Stitt TN, Yancopoulous GD \& Glass DJ (2001) Mediation of IGF1-induced skeletal myotube hypertrophy by $\mathrm{PI}(3) \mathrm{K} / \mathrm{Akt} / \mathrm{mTOR}$ and PI(3)K/Akt/GSK3 pathways. Nature Cell Biology 3, $1009-1013$.

Sakamoto K, Aschenbach WG, Hirshman MF \& Goodyear LJ (2003) Akt signaling in skeletal muscle: Regulation by exercise and passive stretch. American Journal of Physiology 285, E1081-E1088.

Saucedo LJ, Gao X, Chiarelli DA, Li L, Pan D \& Edgar BA (2003) Rheb promotes cell growth as a component of the insulin/TOR signalling network. Nature Cell Biology 5, 566-571.

Tee AR, Fingar DC, Manning BD, Kwiatkowski DJ, Cantley LC \& Blenis J (2002) Tuberous sclerosis complex-1 and -2 gene products function together to inhibit mammalian target of rapamycin (mTOR)-mediated downstream signaling. Proceedings of the National Academy of Sciences USA 99, 13571-13576.

Tipton KD, Rasmussen BB, Miller SL, Wolf SE, Owens-Stovall SK, Petrini BE \& Wolfe RR (2001) Timing of amino acidcarbohydrate ingestion alters anabolic response of muscle to resistance exercise. American Journal of Physiology 281, E197-E206.

Welsh GI, Miller CM, Loughlin AJ, Price NT \& Proud CG (1998) Regulation of eukaryotic initiation factor eIF2B: glycogen synthase kinase-3 phosphorylates a conserved serine which undergoes dephosphorylation in response to insulin. FEBS Letters 421, 125-130.

Zhang Y, Gao X, Saucedo LJ, Ru B, Edgar BA \& Pan D (2003) Rheb is a direct target of the tuberous sclerosis tumour suppressor proteins. Nature Cell Biology 5, 578-581. 